\title{
Evaluating seismic retrofitting efficiency through ambient vibration tests and analytical models
}

\author{
Yves Reuland, Angelo Garofano, Pierino Lestuzzi, lan F.C. Smith \\ Swiss Federal Institute of Technology (EPFL), Lausanne, Switzerland
}

Contact: yves.reuland@epfl.ch

\begin{abstract}
Economic and environmental imperatives lead to an ever growing need to extend the service life of the existing building stock without putting the users at risk. In zones prone to moderate seismic hazard, many buildings were built without considering seismic actions. The design and assessment of efficient seismic retrofitting rely on physical models of the buildings. However, model errors resulting from simplifications and other assumptions might lead to a biased and thus unreliable diagnosis. Therefore, structural measurements are interpreted to reduce the uncertainty related to the ambiguous task of inferring the real structural response of existing buildings, even in the linear elastic range.

This contribution includes the assessment of the retrofitting of an existing masonry building through ambient vibration field measurements. Measured frequencies and mode shapes are interpreted using an error-domain model-falsification framework that allows explicit representation of uncertainties related to modelling and measurement errors. A simple continuous Timoshenko cantilever beam, characterizing the linear elastic dynamic response of the building, is used to model the building. It is concluded that such interpretation of ambient vibration data is useful to assess the efficiency of seismic retrofitting.
\end{abstract}

Keywords: structural identification, model falsification, ambient vibrations, seismic retrofitting, Timoshenko beam, multiple model reasoning

\section{Introduction}

Large parts of the building stock in regions that are prone to moderate seismic hazard were built without considering seismic actions at the design stage. If important infrastructure fails to satisfy minimum seismic capacity demands that have been established by recent codes and regulations, seismic retrofitting is needed.

Dynamic properties of a structure depend on the ratio of mass to stiffness, therefore strengthening has to be carefully designed and performed in order to meet the desired objectives of retrofitting. However, the behaviour of existing structures is subjected to important uncertainties that are related to aspects such as material properties, boundary conditions and the nature of connections between structural elements. These uncertainties may result in unreliable models and therefore, in these situations the use of structural measurements may be useful.

Ambient vibration measurements have been found to carry valuable information on the linear elastic range of dynamic response [1]. Therefore, ambient vibrations constitute an effective source of structural data to assess the efficiency of seismic retrofitting of buildings [2]. In addition, ambient vibration measurements are an attractive 
measurement since they are non-destructive and relatively easy and inexpensive.

The increase in precision and accessibility of measurement devices that has been observed in recent years has resulted in an increasing interest in measurement interpretation methodologies that are suitable for large full-scale engineering structures.

Error-domain model falsification (EDMF) is a structural identification methodology that relies on a basic concept of scientific discovery [3].The measured behaviour of a structure allows falsification of incompatible model predictions rather than the validation of a single model producing compatible results [4].

Even the most sophisticated model is an idealized representation of an open-world structure and thus, model errors are present. The complete model error, including form and spatial correlations of many sources of model errors cannot be known precisely in most structures [5]. Therefore, EDMF uses engineering heuristics to estimate uncertainty bounds considering the type of model used as well as simplifications and other assumptions.

Although the use of EDMF has been assessed for several types of measurement interpretation, including ambient vibrations [6], mode-shapes have never been explicitly included to falsify inaccurate model instances.

The representation of buildings of moderate height by a continuous cantilever beam has been used in the past for regular geometries [7]. The linear dynamic behaviour of a building is therefore simply described by a Timoshenko beam model.

However, past comparisons of Timoshenko beammodels with measured dynamic data relied on fine-tuning the frequency predictions by updating single-valued parameters, such as the Young's modulus [8]. A multiple-model reasoning framework, such as model falsification, providing ranges of prediction rather than single answers, has not been combined with Timoshenko beam models of building behaviour.

This contribution presents the application of error-domain model falsification to a four-storey building that has been retrofitted to meet requirements in a moderate seismic zone in Switzerland. Frequencies and mode shapes determined from field measurements of ambient accelerations are used to reduce the uncertainty related to the linear dynamic response of the building.

\section{Methodology}

Assessing the structural behaviour of existing structures is an ambiguous task that can be undermined by large uncertainties concerning boundary conditions and parameter values among others. Therefore, several structural identification techniques, defined here as the task of comparing analytical model predictions with measured quantities of the real structure, have emerged to enable better insights into the behaviour of existing structures.

\subsection{Error-domain model falsification}

Error-domain model falsification builds upon the premise that measured evidence is best used to falsify inappropriate model instances rather than validate single models. Thus, EDMF starts from a population of model instances that are generated by a grid sampling of the parameter space. The possible parameter ranges are derived from engineering knowledge.

A physical model $\mathrm{g}($.$) is used to predict the$ structural behaviour from the $n_{p}$ parameters $\theta_{i}$ characterizing the geometry and the materials of the structure and the nature of its boundary conditions.

Provided the right parameter values $\theta^{*}$ are used, the model prediction $g\left(\theta^{*}\right)$ returns the 'real value' of a structure flawed by a model error $\varepsilon^{*}{ }_{\text {model }}$. Similarly, the measured quantity y corresponds to the real value flawed by a measurement error $\varepsilon^{*}$ measurement. Hence, the fundamental equation of error-domain model falsification states for each measure or prediction $i$ :

$$
g_{i}(\boldsymbol{\theta})+\epsilon_{\text {model }}^{*}=\boldsymbol{y}_{i}+\epsilon_{\text {measurement }}^{*}
$$

In reality however, neither the measurement error, nor the model error can be exactly known. Thus, engineering knowledge is used to identify potential sources of modelling and measurement 
errors. Based on this heuristic knowledge reasonable bounds to the uncertainty on the errors are estimated.

By combining the estimations of the many sources of uncertainties, a distribution of the residual between model prediction and measurement can be computed. Based on this distribution and a given target probability $\varphi$, thresholds can be defined. According to Equation 2, a model instance is falsified if, for any of the $n_{m}$ measurements, it lies outside of the thresholds.

$$
\begin{gathered}
T_{\text {low }, i}\left(\varphi^{\frac{1}{n_{m}}}\right) \leq g_{i}(\theta)-y_{i} \leq T_{\text {high }, i}\left(\varphi^{\frac{1}{n_{m}}}\right) \\
\forall i \in\left\{1, n_{m}\right\}
\end{gathered}
$$

When two mode-shapes are compared, the Modal Assurance Criterion (MAC) is computed as the analogue to the residual between measurement and model. The expression of the MAC between two vectors is recalled in Equation 3. The MAC between two orthogonal vectors equals 0 and between two collinear vectors equals 1 .

$$
\operatorname{MAC}(\boldsymbol{u}, \boldsymbol{v})=\frac{\left|\boldsymbol{u}^{t} \boldsymbol{v}\right|^{2}}{\left|\boldsymbol{u}^{t} \boldsymbol{u}\right|\left|\boldsymbol{v}^{t} \boldsymbol{v}\right|}
$$

Therefore, thresholds that allow the falsification of models based on the mode shape are expressed as a MAC value. The MAC threshold is derived from a stochastic combination of the uncertainty on the measured mode shape $\varepsilon_{\text {MAC,measured }}$ and the predicted mode shape $\varepsilon_{\text {MAC,model. }}$

The measurement uncertainty for the mode

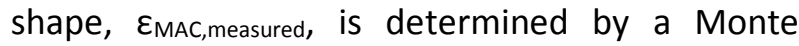
Carlo combination of the MAC between the vector containing the measured modal displacements $y_{k}$ and a vector of the measured mode shape plus random instances of the variable describing the measurement uncertainties, $\mathrm{y}_{\mathrm{k}}+\varepsilon_{\mathrm{MAC} \text {,measured. }}$

In a similar way, the model uncertainty related to the mode shape is obtained from the MAC between the mean model prediction $g(\bar{\theta})$ and the mean model prediction plus the model error $\varepsilon_{\text {model, }}$ like stated in equation (3).

$$
\varepsilon_{M A C, \text { model }}=\operatorname{MAC}\left(\boldsymbol{g}(\overline{\boldsymbol{\theta}}), \boldsymbol{g}(\overline{\boldsymbol{\theta}})+\boldsymbol{\varepsilon}_{\text {model }}\right)
$$

The total uncertainty related to the mode shape is then obtained by combining the MAC- uncertainties originating respectively from the measurement and from the model respectively.

A model instance is accepted to be a candidate model if the MAC between the measured mode shape and the predicted one exceeds the determined MAC threshold. Therefore, when mode shapes are used in the error-domain model falsification, Equation (2) becomes, for mode k:

$$
\operatorname{MAC}\left(\boldsymbol{g}_{\boldsymbol{k}}(\boldsymbol{\theta}), \boldsymbol{y}_{\boldsymbol{k}}\right) \geq T_{M A C}\left(\varphi^{\frac{1}{n_{m}}}\right)
$$

The number of measurements $n_{m}$ is obtained from the sum of quantities compared with equation (2) that applies to static measurements or natural frequencies and mode shapes compared via equation (4).

A whole model class $\mathrm{g}($.$) is falsified, if all the$ model instances lie outside of the threshold bounds for one of the measured quantities. A falsified model class is an indication of wrong model assumptions; examples are incorrect boundary conditions and ignoring phenomena such as cracking.

To be consistent with the framework of multiple model reasoning, all remaining candidate models are considered equally probable. Therefore, predictions are carried out using all candidate models plus the model uncertainty that has been estimated for a given model class.

\subsection{Timoshenko beam model}

The horizontal modal deformations of a Timoshenko beam model originate from two contributions: the bending motion and the shear motion. The dimensionless parameter $\mathrm{C}$ characterizes the nature of the Timoshenko beam as a function of bending stiffness El and the shear stiffness K. For a cantilever beam, clamped at the bottom and of height $\mathrm{H}$, the parameter $\mathrm{C}$ is given by:

$$
C=\frac{\pi^{2} E I}{4 K H^{2}}
$$

When $C$ takes a value of 0 , the Timoshenko beam degenerates to a Bernoulli beam. For high values of $C(C \rightarrow \infty)$ the beam behaves like a pure shear beam. 
Defining $m_{L}$ as the linear mass of the beam, the translation motion $U(x)$ is then given by the differential equation (2).

$$
E I U^{(4)}(x)+\omega^{2} \frac{E I}{K} m_{L} U^{(2)}(x)=\omega^{2} m_{L} U(x)
$$

With $\delta_{1 \mathrm{k}}$ being a discrete root (close to the odd integer sequence) that can be determined for a given value of $\mathrm{C}$, the $\mathrm{k}$-th frequency of a Timoshenko beam with clamped-free boundary conditions is determined from equation (3).

$$
f_{k}=\frac{1}{4 H} \sqrt{\frac{K}{m_{L}}} \frac{\sqrt{C} \partial_{1 k}^{2}}{\sqrt{1+C \partial_{1 k}^{2}}}
$$

The mode-shape predictions of a Timoshenko beam model only depend on the value of $C$.

\section{Case study}

This methodology is applied to a school building in Switzerland that has been transformed and retrofitted in order to meet new seismic requirements.

In the initial configuration, the five-storey building was characterized by masonry walls and wooden slabs. During the transformation, the wooden slabs were replaced by concrete slabs, and several well anchored concrete walls were added. A scheme of the transformation can be found in figure 1 .

The storey height of the building is $3 \mathrm{~m}$ and the geometry of the building is close to identical between storeys. The basement of the building is considerably stiffer than the remaining floors, therefore the basement is omitted from the analysis.

\subsection{Ambient vibration measurements}

Ambient vibrations have been recorded using 6 tri-dimensional Epi-Sensor accelerometers (Kinemetrics). Acceleration time series were recorded for four sensor configurations, thereby covering a total of 16 sensor locations in addition to a common reference point. This resulted in eight sets of acceleration data of a duration of 10 minutes and a sampling frequency of $200 \mathrm{~Hz}$.

The measurements on the upper floor have been rejected because the sensor could not be placed directly on the floor and thus, the record characterizes the local behaviour of the secondary elements. Consequently, comparisons between measured and modelled mode shape are made for the first three floors.

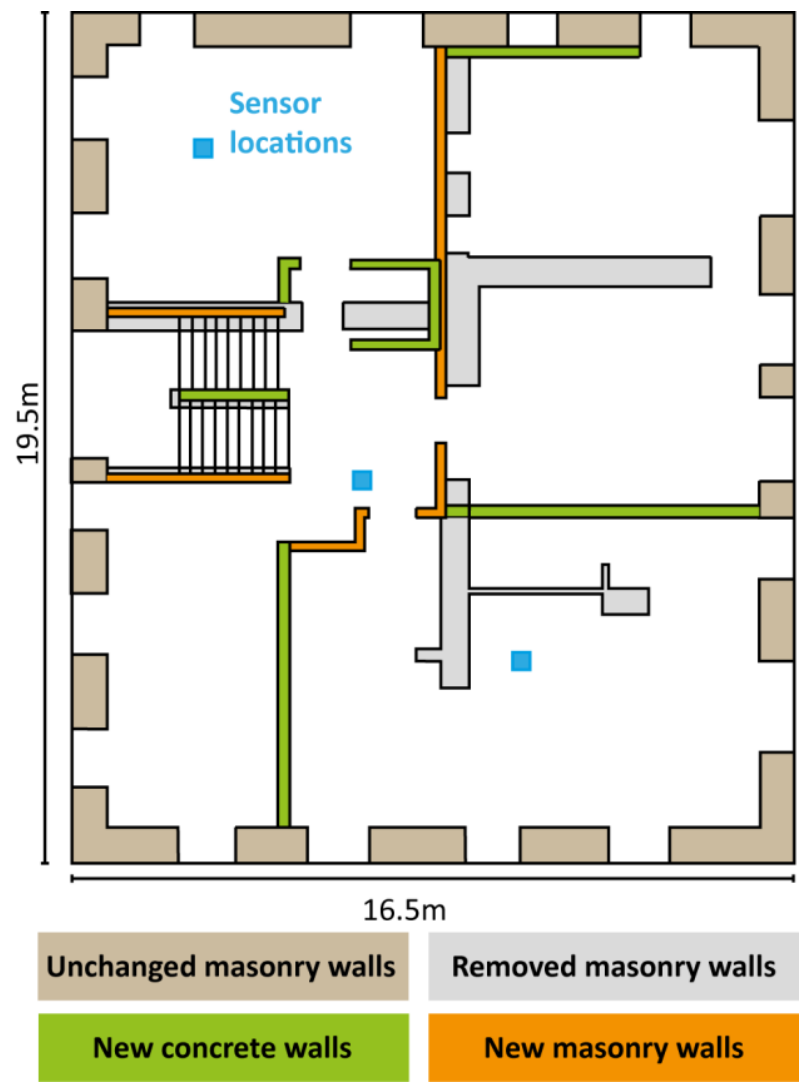

Figure 1 Scheme of the studied building and the transformation activities

Given the simplicity of the model that is used to predict the structural behaviour, the vertical components of the measurement are ignored.

The dynamic properties of the building are derived using the Frequency Domain Decomposition that has been well established for similar applications [9].

The natural frequencies are determined by the peaks in the plot of the singular values of the correlation power spectral density (CPSD). If the second singular value has a peak near the peak of the first singular value, this indicates the presence of close orthogonal modes.

Figure 2 presents the singular value plot of the CPSD matrix for the measurement set related to 
the upper floors (for the sensor positions, see Figure 1).

The post-transformation plot shows the peak related to the first longitudinal mode $\left(f_{1}\right)$ on the first singular value and the peak related to the first transversal mode $\left(f_{2}\right)$ on the second singular value. However, on the pre-transformation plot, both modes lie on the first singular value. The peak on the second singular value is related to a combined mode and partly to the longitudinal mode in the frequency range where the transversal mode dominates the first singular value.

The peaks corresponding to the first frequencies in the two main directions do not correspond to exactly the same frequency over the eight data sets. This can partly be explained by the nonstationary nature of ambient vibrations and measurement noise.

The frequencies used for the subsequent falsification process are obtained by averaging the peaks identified over the various data sets and are summarized in Table 1.

Table 1. Identified natural frequencies

\begin{tabular}{ccc}
\hline State & $\begin{array}{c}\mathbf{f}_{1} \\
{[\mathbf{H z}]}\end{array}$ & $\begin{array}{c}\mathbf{f}_{\mathbf{2}} \\
{[\mathbf{H z}]}\end{array}$ \\
\hline Initial & $\begin{array}{c}4.31 \\
(\sigma=0.04)\end{array}$ & $\begin{array}{c}4.52 \\
(\sigma=0.05)\end{array}$ \\
\hline Transformed & $\begin{array}{c}6.15 \\
(\sigma=0.03)\end{array}$ & $\begin{array}{c}6.27 \\
(\sigma=0.14)\end{array}$ \\
\hline
\end{tabular}

The uncertainty on the natural frequencies is also reflected in the determination of the measured mode shape. However, by averaging the modal displacements per floor, and by isolating the two main directions this uncertainty can be reduced.

Considering only the components of the main direction of a given mode is justified in this case, because the positions related to the frequency uncertainty lie inside the MAC-bell. The MAC-bell is defined to cover the frequencies for which the mode corresponding mode shape has a MAC value of more than 0.8 with the mode shape corresponding to the natural frequency identified for a given set. In addition, the Timoshenko beam model used to predict the frequencies and mode shapes is separated for the two directions.

\subsection{Continuous beam model}

The dynamic properties of the building have been predicted using a continuous Timoshenko beam model. The material properties used to build the model population are summarized in Table 2 .

The values for the Timoshenko parameter correspond to the transition between a BernoulliEuler beam and a pure shear beam. These values represent the extremes and therefore explain the predominant influence of this parameter on the predictions. In addition, it has to be noted that the mode shape is independent on the parameters other than C.

Several sources of uncertainty related to the ambient vibration measurements have been identified and their bounds estimated:

- The precision of accelerometers is estimated be prone to a relative zero-mean Gaussian uncertainty of $1.5 \%$ for the frequencies and $3 \%$ for the modal displacements.

- The Fourier transform that is used to transfer the acceleration time series into the frequency domain is estimated to induce a relative zeromean Gaussian errors of $2 \%$ standard deviation on frequencies and mode-shape components.

- The varying positions of the frequency peaks for the different time sets is estimated to produce a native zero-mean Gaussian error on the frequency of $0.15 \mathrm{~Hz}$ (cf. Table 1) and of 0.01 on the MAC.

- A potential lack of synchronization between the recordings of the accelerometers is accounted for with an uncertainty of $0.2 \%$ on the MAC [10].

- The secondary parameters that have a relative importance below five percent (i.e. density of concrete, density of wood and the Poisson's ratio for concrete and masonry) are used to predict the model uncertainty related to the omission of these parameters from the analysis.

The continuous beam model that has been used to model the structure is subject to important simplifications. Given the idealized and unrealistic clamped boundary conditions, the omission of the contribution of spandrels to the stiffness and the neglecting of secondary elements, the estimation 
of the model error is a biased uniform relative

Prior to transformation

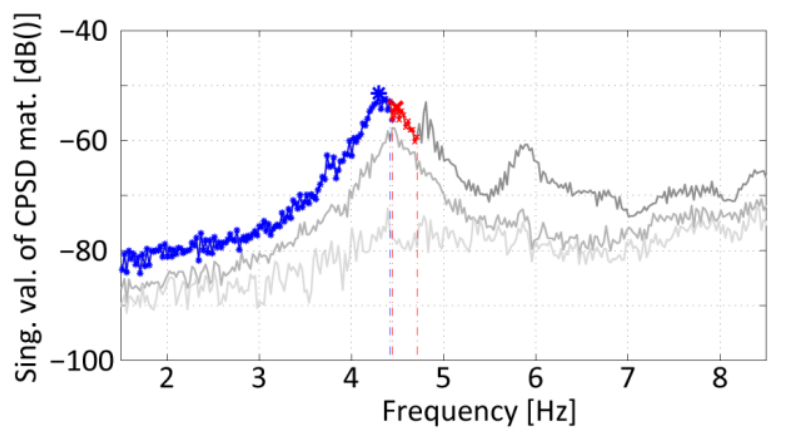

-1st singular value _ 2 nd singular value _ 3 rd singular value distribution between $-15 \%$ and $+7.5 \%$.

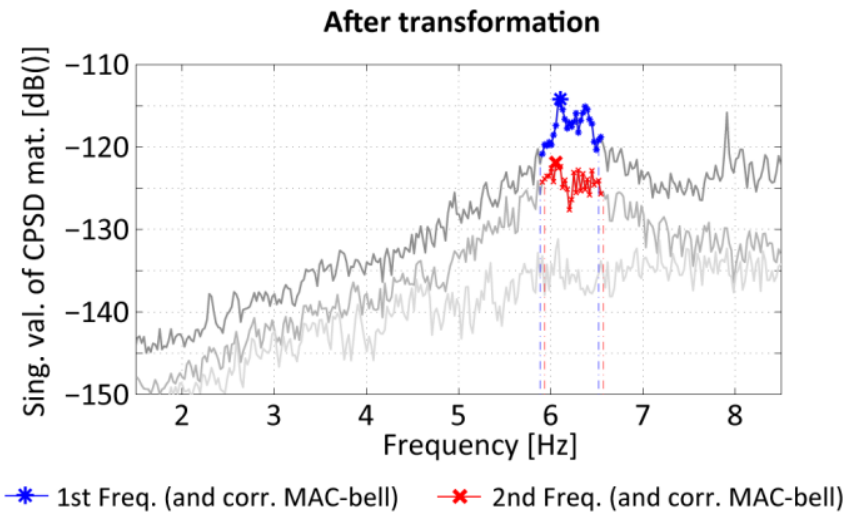

Figure 2. First three singular values of the correlation power spectral density (CPSD) matrix for the frequency Domain Decomposition of ambient vibration records performed before respectively after the transformation

This means that the model is assumed to be more likely to overestimate the stiffness (mainly due to the boundary conditions). Error-domain model falsification allows explicit use of a biased uncertainty.

\subsection{Results of model falsification}

Error-domain model falsification is performed using the first frequencies in both directions and their respective mode shape.

Figure 3 shows the model falsification based on the first longitudinal frequency. Candidate models are models that lie inside the uncertainty thresholds for the four considered measurements.

The MAC criterion used to falsify models based on the mode shape is presented in Figure 4. Predictions lie on horizontal lines because modal displacement predictions based on a continuous Timoshenko beam depend on the only parameter $C$, characterizing the nature of the beam.

Given the maximum MAC value of 1 , only a lower threshold exists and the uncertainty distribution has a unilateral tail.

In the initial state, 339 of 360 model instances have been falsified. In the retrofitted state, 2507 of 2700 model instances have been falsified. Given the relative importance of the parameters (Table 2 ) it is not surprising that the most notable reduction in parameter ranges is obtained for the $C$ parameter. The identified range for C corresponds to $0.09-2.4$ for the initial state and to 0.58-30 for the retrofitted state.

Table 2. Parameter ranges and relative importance for the Timoshenko beam model

\begin{tabular}{ccc}
\hline Parameter & Range & $\begin{array}{c}\text { Relative Importance } \\
\text { (Initial/Transformed } \\
\text { state) }\end{array}$ \\
\hline $\begin{array}{c}\text { Young's } \\
\text { modulus } \\
\text { masonry [GPa] }\end{array}$ & $5-15$ & $26 \% / 16 \%$ \\
\hline $\begin{array}{c}\text { Timoshenko } \\
\text { parameter C [-] }\end{array}$ & $0.003-30$ & $63 \% / 72 \%$ \\
\hline $\begin{array}{c}\text { Density } \\
\left.\text { masonry [t/m }{ }^{3}\right]\end{array}$ & $1.2-2.0$ & $11 \% / 6 \%$ \\
\hline $\begin{array}{c}\text { Young's } \\
\text { modulus } \\
\text { concrete [GPa] }\end{array}$ & $25-40$ & $-/ 6 \%$ \\
\hline
\end{tabular}

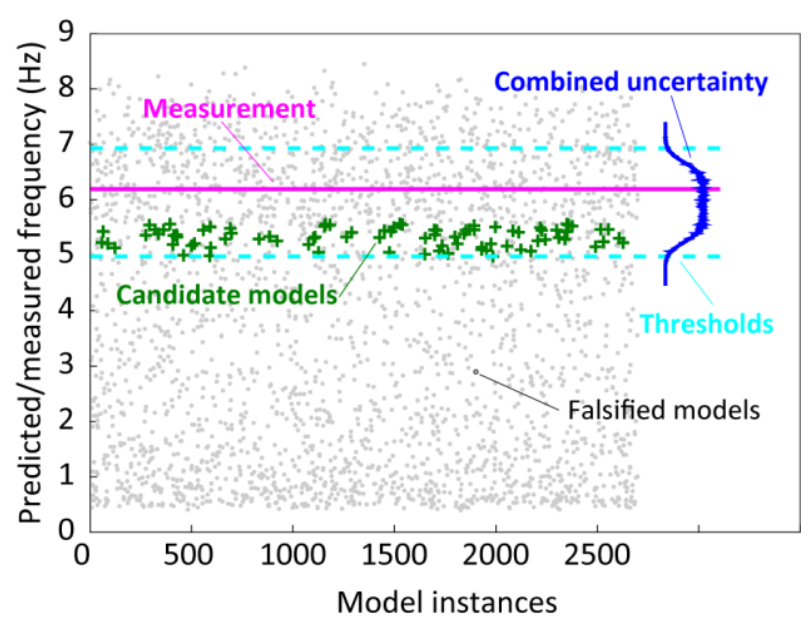

Figure 3. Falsified and accepted model instances related to the first longitudinal frequency 
The first longitudinal frequency corresponding to the identified candidate models has been used to predict the displacement demand from the spectrum (Micro-Zone S9, Rhone valley). This diagnosis is obtained by combining the predictions corresponding to the candidate models with the model error.

As can be seen in Figure 5, the estimate of the spectral displacement demand is reduced, thus it is concluded that the seismic retrofitted is effective.

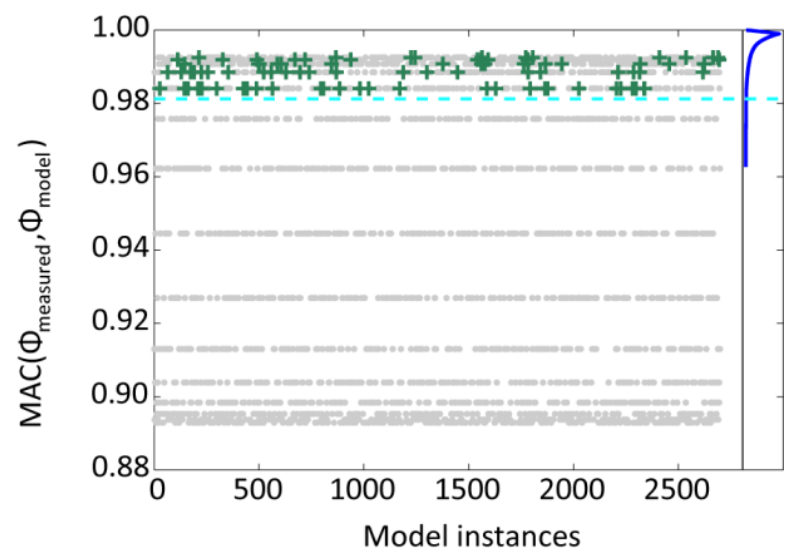

Figure 4. Falsification based on the first longitudinal mode-shape

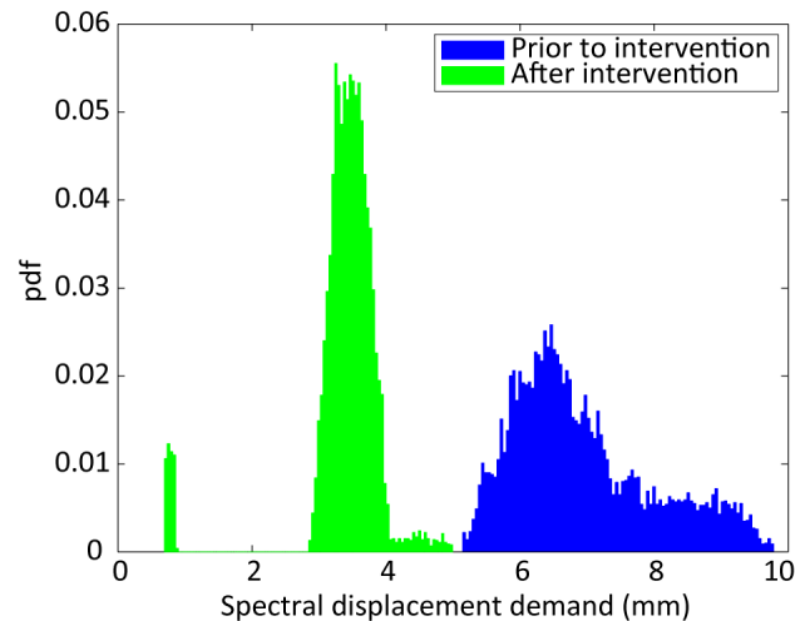

Figure 5. Comparison of the distribution of spectral displacement demand before and after retrofitting

\section{Discussion}

The diagnosis of the building in its initial and strengthened state leads to the conclusion that the seismic retrofitting has successfully reduced the displacement demand of the building. However, this is related to an increase of force that the building has to withstand. Nonetheless, the supplementary reinforced concrete elements that have been added are a sufficient reinforcement to bear supplementary forces.

In order to draw further conclusions regarding the efficiency of the connection between the new slabs and the existing walls as well as the redistribution of forces between the structural elements in a mixed concrete-masonry building, a more complex model of the structure is needed.

In addition, the analysis is based on ambient vibrations, characterized by low excitations, typically between 0.1 and $100 \mu \mathrm{m} / \mathrm{s}^{2}$. Thus, frequency is representative of the linear elastic structural response only. Although the real frequency related to higher excitation amplitudes will be lower than the frequency characterizing the ambient vibration response, approximate relationships between the two frequencies are available [11]. Also, it is assumed that the retrofit will remain at least equally sound as the rest of the structure during an earthquake.

The principal aims of this contribution are to perform structural identification and then compare states before and after the intervention. Therefore, the displacement demand based on the elastic frequency can be used as criterion for comparing the two states.

\section{Conclusions}

This contribution on structural identification of an existing building based on ambient vibrations leads to the following conclusions:

- The error-domain model-falsification methodology explicitly includes values of model uncertainty for determination of ranges of possible values of parameters that determine dynamic response of a building, and therefore, it is a valuable tool for assessing vulnerability with respect to future earthquake actions.

- Use of error-domain model falsification permits many sources and types of uncertainty to be taken into account, and this makes it attractive for the interpretation of ambient vibration data. 
- Mode-shape measurements have potential to falsify model instances of a continuous Timoshenko beam model, and thus they contribute to reducing the uncertainty related to the linear-elastic dynamic response of existing structures.

\section{Acknowledgements}

This paper summarizes part of a longer article submitted to Journal of Structural Engineering. The authors would like to acknowledge the municipal authorities of Bex as well as Ingex SA for allowing and facilitating measurements. Costs of the measurement system were partially covered by the Swiss National Science Foundation under grant number 150785. Finally, Amin Karbassi and Einar Gudmundsson are thanked for their contributions to the vibration measurements.

\section{References}

[1] Michel C, Gueguen P, Causse M. Seismic vulnerability assessment to slight damage based on experimental modal parameters. Earthquake Engineering \& Structural Dynamics. 2012; 41(1):81-98.

[2] Karbassi A, Michel C, Lestuzzi P. Ambient vibration tests and numerical modelling to evaluate seismic retrofitting. Proceedings of the Second Eur. Conf. on Earthquake Engineering and Seismology. 2014

[3] Popper KR. The logic of scientific discovery. London: Hutchinson. 1959;

[4] Raphael B, Smith I. Finding the right model for bridge diagnosis. Artificial intelligence in str. Eng., Springer; 1998. p. 308-19.

[5] Goulet J-A, Smith IFC. Structural identification with systematic errors and unknown uncertainty dependencies. Computers \& Structures. 2013; 128:251-8.

[6] Goulet J-A, Michel C, Smith IF. Hybrid probabilities and error-domain structural identification using ambient vibration monitoring. Mechanical Systems and Signal Processing. 2013; 37(1):199-212.
[7] Michel C, Hans S, Guéguen P, Boutin C. In situ experiment and modelling of RCstructure using ambient vibration and Timoshenko beam. 1st European Conference on Earthquake Engineering and Seismology (ECEES). 2006

[8] Boutin C, Hans S, Ibraim E, Roussillon P. In situ experiments and seismic analysis of existing buildings. Part II: Seismic integrity threshold. Earthquake Engng Struct Dyn. 2005 Oct 1; 34(12):1531-46.

[9] Brincker R, Zhang L, Andersen P. Modal identification of output-only systems using frequency domain decomposition. Smart Mater Struct. 2001 Jun 1; 10(3):441.

[10] Krishnamurthy V, Fowler K, Sazonov E. The effect of time synchronization of wireless sensors on the modal analysis of structures. Smart Mater Struct. 2008; 17(5):055018.

[11] Michel C, Zapico B, Lestuzzi P, Molina FJ, Weber F. Quantification of fundamental frequency drop for unreinforced masonry buildings from dynamic tests. Earthquake Engineering \& Structural Dynamics. 2011; 40(11):1283-96. 